\title{
Subject Index Vol. 39, 1995
}

\section{Acetic acid 107}

Adult males 177

Age 16,117

Alkaline phosphatase 107

Amino acids 317

Anorectic agents 107

Antioxidant 99

- $\quad$ vitamins 63, 208

Ascorbic acid 208, 217, 227, 310

Bioelectrical impedance 234,242 Blood cholesterol 291

glucose, exercise 203

plasma, fluorometric riboflavin titration 355

Body build 234

composition 177

-, analytical methods 325

fat 325

potassium 177

- $\quad$ water 325

Brain 117

Bromide-dilution method 242 Butylated hydroxytoluene 99

13Clabeling 143 Cadmium 361 Caffeine 135 Calf 164

Carbohydrates 251 ß-Carotene 334 Carotenes 208 Cell migration 1

- $\quad$ proliferation 1

Cell-mediated immune response

296 Chicken 302 Chicks 107 Cholecalciferol 227 Cholesterol 71

secretion 185 Cirrhosis 346 Colon 42 Cooking 16 Copper 271,334

metabolism 227

Coronary heart disease 52 Couscous 310 Cycas 42

Delayed hypersensitivity reaction

$296 \Delta 6$ desaturase 117 Diabetes 217 Diet 42,52,291 Dietary fat 193

fish 261

glucose 143

starch 143 Dietary vegetable oil 261 Digestive enzyme 164 Duplicate diet 208

Dutch National Food Consumption Survey 85 Dyslipidemia 217

Eating behavior 371 Elderly 371 Encephalomalacia 302 Endothelial cell injury 1

cells 1 Energy 42,371

expenditure 135

intake 271 Enzyme 42

Erythrocyte membranes 63 Essential fatty acid(s) 36 
- - deficiency 9 Ethanol ingestion 152 Extracellular water 177,234

-, validation 242

Fat 271

Fat-free mass 325

Fat-soluble vitamins 124

Fatty acids 117,302

59Fe absorption, rat 310

Feed restriction 107

Flavin adenine dinucleotide 224

- mononucleotide 224

Fluorometry, riboflavin titration

355

Fluoxetine 159 Fortification 85 Free amino acids pools 16

- $\quad$ fatty acids 71

Gastric ulcers 285

Genetics 285

Geriatric patients, malnutrition

340 German children, energy intake

271 Glutathione 28 Glycerol, oral 71 Glycogen 143 Glycoprotein 143 Goitre prophylaxis 85

Growth hormone-deficient adults

242 GTP activity 95

High-performance liquid chroma-

tography 224 High-protein diet 152 Human blood plasma 224

- $\quad$ skin condition 9

Humans 291

ß-Hydroxybutyrate 107

Insufficient vitamin intake, risk 124

Insulin-dependent diabetes mellitus 217

Internal temperature 135

Intestinal macromolecular absorption 152

- $\quad$ resection 227

Intestine 143

Intracellular water 177

Iodine intake 85

Iron bioavailability 310

- $\quad$ overload 63

Ketone body production 185

Lard fat 193 Lentil 16

382

Leucine 346 Leydig cell count 95 Lipids 42 Lipoprotein(s) 291

secretion 63 Liver 117,346

glycogen 203 Long-chain polyunsaturated fatty

acids 36

- $\quad$ triglycerides 251

Low fat intake 124

Magnesium 217,285 Medium-chain triglycerides 251 Microbial phytase 361 Microflora 42 
Microsomal lipid peroxidation 99 Mineral, trace element bioavailability 361 Mouse 285 mRNA 164

Multifrequency impedance 177 Muscle composition 317 -, liver 279 Myocardial infarction 52 n-6 and n-3 fatty acids 251 Non-insulin-dependent diabetes

mellitus 217 Normal Japanese 261 Nutrition policy 85 Nutritional questionnaire, elderly 340

- $\quad$ risk assessment, geriatric

patients 340

Obesity 159 (8-14C)-Octacosanol 279 Olive oil 193 Overweight/obesity 371 Oxidation

resistance 63 Oxidized lipoproteins 1

Paediatric nutrition 36

Pancreas 164

Partially hydrogenated fats 36

Pea 164

Perfused liver 185

Phospholipase A2 302

Phyticacid 361

Phytosterols 291

Placebo 159

Plasma arachidonic acid 261

docosahexaenoic acid 261

eicosapentaenoic acid 261

lipid composition 52 Pregnancy 28 Prescholers 124 Probucol 99 Proliferation 42 Propionic acid 107 Protein 271

malnutrition 152

metabolism 317,346

restriction 28 Pyridoxine 285

Rat 16,28 Repletion 95 Retinol 208 Retinyl palmitate 71 Riboflavin 224

- $\quad$ status 355

Riboflavin-binding apoprotein

355 Ribonucleic acid 143

Salbutamol 317 Selenium 334 Serial dose 279 Serum cholesterol 99

fatty acids 193

lipids 9,193

phospholipids 251 Simulation study 85

Skin temperature 135 Soya bean 164 Sperm count 95 Sri Lanka 9 Stress 285 Sucrose 203

Sunflower oil 193

Tea decoction 310 Tissue distribution $279 \alpha$-Tocopherol 99, 302 Tocopherols 208 Total body water 177,234

parenteral nutrition 251 Trace elements 334 Trans-fatty acid 36, 185 Trauma 251 Treatment 217

Triglyceride(s) 71

secretion 185 Triton WR 133963 Tropical sprue 296

Vegetarians 334 VFA 107 Vitamin(s) 334

A 334

- deficiency 9

B2 355 


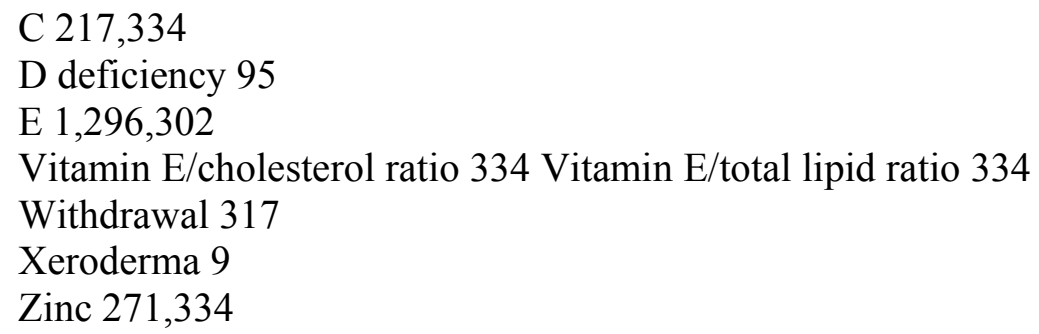

